\title{
Il punto scritto: genesi e scrittura ne Il sorriso dell'ignoto marinaio
}

\section{Daragh O'Connell}

\section{(2) OpenEdition}

1 Journals

Edizione digitale

URL: https://journals.openedition.org/cher/1177

DOI: $10.4000 /$ cher. 1177

ISSN: 2803-5992

\section{Editore}

Presses universitaires de Strasbourg

\section{Edizione cartacea}

Data di pubblicazione: 6 novembre 2018

Paginazione: 55-76

ISBN: 979-10-344-0027-0

ISSN: 1968-035X

Notizia bibliografica digitale

Daragh O'Connell, «ll punto scritto: genesi e scrittura ne // sorriso dell'ignoto marinaio», reCHERches

[Online], 21 | 2018, online dal 05 octobre 2021, consultato il 19 novembre 2021. URL: http://

journals.openedition.org/cher/1177 ; DOI: https://doi.org/10.4000/cher.1177 


\title{
Il punto scritto: genesi e scrittura ne Il sorriso dell'ignoto marinaio
}

\author{
Daragh $0^{\prime}$ Connell $^{1}$
}

$\mathrm{I}^{\mathrm{n}}$ n un'intervista fatta a Vincenzo Consolo parecchi anni fa, lo scrittore siciliano ha parlato della "fantasia creatrice" come di un elemento femminile con il quale si può uscire dal cerchio della ragione, un cerchio simboleggiato dalla metafora della "chiocciola" nel Sorriso dell'ignoto marinaio (O’Connell, 2004: 238-253). Questa fantasia creatrice nel romanzo, si muove nei panni del personaggio Catena Carnevale la fidanzata che sfregia il sorriso ironico e pungente del ritratto e, in seguito, ricama la tovaglia di seta intitolata «L'albero delle quattro arance». La descrizione nel libro di questa tovaglia è fondamentale e assomiglia per certi versi proprio allo stile della scrittura consoliana, ed è in realtà una metafora per il testo stesso, se non l'intero progetto letterario di Consolo. Leggiamo:

Sembrava, quella, una tovaglia stramba, cucita a fantasia e senza disciplina. Aveva sí, tutt'attorno una bordura di sfilato, ma il ricamo al centro era una mescolanza dei punti più disparati: il punto erba si mischiava col punto in croce, questo scivolava nel punto ombra e diradava fino al punto scritto. E i colori! Dalle tinte più tenui e sfumate, si passava d'improvviso ai verdi accesi e ai rossi più sfacciati. Sembrava, quella tovaglia, - pensò la baronessa, - ricamata da una invasa dalla furia, che con intenzione ha trascurato regole numeri misure e armonia, fino a sembrare che la ragione le fosse andata a spasso. (Consolo, 2015: 167-168)

Questa analogia tra la scrittura letteraria e il ricamo e lo sfregio di Catena, richiama proprio la poetica distintiva di Consolo: una poetica palinsestica che prevede l'accumulo e l'elisione di vari testi di provenienza diversa, siano essi di stampo giornalistico, creativo o saggistico, in uno spazio di singolare gestazione autoriale fra polifonia e palinsesto (O’Connell, 2008: 161-184).

Queste pagine intendono analizzare, da diversi punti di vista, il primo capitolo del longseller di Vincenzo Consolo, Il sorriso dell'ignoto marinaio (1976), delineando l'evoluzione del romanzo dalla sua forma primigenia fino

1 Daragh O’Connell, University College Cork. 
alle più recenti rielaborazioni. Saranno messe a fuoco alcune varianti testuali (Messina, 2009: 143-202) e dichiarate le fonti, letterarie e non, sottese all'intero capitolo. Il primo capitolo è in effetti, a mio avviso, un grande palinsesto, e molte delle procedure adottatevi da Consolo richiamano una polifonia e una concezione alla Bachtin dell'enciclopedismo, la sua definizione di romanzo di Seconda linea. In più, il capitolo I sancisce in realtà molti aspetti della nuova poetica di Consolo, che in definitiva rappresenta lo spazio letterario in cui il passaggio da riso a sorriso è più evidente.

Consolo conclude così la Nota dell'autore, vent'anni dopo aggiunta come postfazione dell'edizione Mondadori del 1997:

[...] che senso ha la riproposta di questo Sorriso? E la risposta che posso ora darmi è che un senso il romanzo possa ancora trovarlo nella sua metafora. Metafora che sempre, quando s'irradia da un libro di verità ideativa ed emozionale, allarga il suo spettro con l'allargarsi del tempo. (Consolo, 1997: 183)²

Sono passati vent'anni da queste enunciazioni, e oggi il testo sfaccettato di Consolo ha cominciato a ricevere il trattamento critico che merita. Abbiamo avuto oltre quarant'anni per metabolizzare e situare un romanzo della sua importanza, non solo nel contesto della narrativa italiana del ventesimo secolo, ma anche in quello dellevoluzione poetica di Consolo. Si dovrebbe, pertanto, entrare nel cuore del dibattito su questo allargarsi del tempo, contribuire attivamente alla crescita di questo corpus critico-accademico con al centro la poetica di Consolo. Tuttavia, in questo approccio al capitolo I, ciò che più ci preme non è tanto il suo allargarsi nel tempo, quanto il suo restringervisi, cercando di individuare con esattezza i momenti cardine della composizione del testo.

Sarebbe erroneo, in tanti sensi interconnessi, dire che Il sorriso è un testo nel fiore dei suoi quarant'anni. Quando leggiamo le mini-biografie sulle copertine dei libri di Consolo, siamo portati a credere che $\mathrm{Il}$ sorriso sia un prodotto testuale della metà degli anni '70 e perfettamente inserito nel suo tempo. In realtà, va sottolineato che $\mathrm{Il}$ sorriso è un testo mutevole, «un lavoro perennemente mobile e non finibile», per dirla con Contini (1970: 5), la cui genesi può essere collocata intorno alla metà degli anni '60 e le cui più recenti articolazioni possono essere datate al 1997. Questo saggio intende dimostrare che Il sorriso si può considerare come un atto letterario il cui incipit - non solo testuale, ma anche concettuale - è retrodatabile a cinquant'anni fa.

Fuori dalla storia del testo in quanto tale, alcune delle tematiche e delle metafore de $\mathrm{Il}$ sorriso sono apparse in diverse guise, in altre narrazioni più tarde di Consolo. Sia Nottetempo, casa per casa sia L'olivo e l'olivastro si riferiscono esplicitamente al Ritratto d'uomo di Antonello (Consolo, 1992a: 138-139 [Consolo, 2015: 142-143]; Consolo, 1994: 124-125 [Consolo, 2015: 852853]). Per cui si può dire che Il sorriso, dal suo livello ipertestuale originario,

2 La Nota dell'autore, vent'anni dopo è successivamente riapparsa come saggio di chiusura in Consolo, 1999a, p. 276-282, con il titolo «Il sorriso», vent'anni dopo. 
è diventato un ipotesto per le opere successive di Consolo ${ }^{3}$. Per di più, $\mathrm{Il}$ sorriso è il primo capitolo di una trilogia di romanzi che prendono le mosse da importanti momenti della storia siciliana e italiana: l'insorgere del fascismo nei primi anni '20 e la sua correlazione in tempi più recenti con la nuova destra italiana, in Nottetempo, casa per casa (1992); e il fallimento del tentativo di creare una società giusta da parte della comunemente definita "generazione del dopoguerra", simbolizzato dall'assassinio del giudice Paolo Borsellino, in Lo spasimo di Palermo (1998).

Tralasciando queste considerazioni, la focalizzazione esclusiva su Il sorriso e, in particolare, sul capitolo iniziale del romanzo, si basa su alcune valide ragioni: anzitutto, il capitolo I risulta il più negletto di tutti, dato che la critica ha preferito studiare e interpretare i cosiddetti "capitoli caldi" della seconda metà del romanzo; poi, perché è il capitolo con la storia testuale più lunga, e dunque la sua gestazione dovrebbe apparire più evidente ad un'attenta analisi; infine, il capitolo I inaugura una nuova poetica per Consolo dopo La ferita dell'aprile (1963): gli elementi di solito associati al romanzo - il significato del sorriso, la chiocciola, la compresenza di poesia e prosa, le forme metriche, gli stilemi, l'uso dell'elencazione, di forme dialettali e parodistiche, l'impegno dell'autore, e un'abbondante messe di intertesti, in breve, una polifonia - sono già tutti presenti nel loro insieme in questo capitolo.

Certo i commenti dell'autore facilitano la lettura del primo capitolo del romanzo, e le riflessioni sono molto rilevanti, non solo per ciò che nascondono, ma anche per ciò che rivelano. Forse, quella più significativa riguardo al Sorriso appare nella Nota dell'autore chiamata in causa all'inizio, in cui, pur a distanza di venti anni, Consolo delinea i tre elementi fondamentali da cui la struttura del romanzo ha preso forma:

I tre elementi allora, la rivolta contadina di Alcàra, i cavatori di pomice di Lipari e il Ritratto d'Antonello reclamavano una disposizione su uno spazio di rispondenze e di senso, in cui il Ritratto stesso, nel suo presumibile percorso da una Messina, già di forte connessione storica, cancellata dai terremoti, a Lipari, isola-regno d'esistenza, di mito, a Cefalù, approdo nella storia e nella cultura, disegnava un triangolo e un movimento da un mare d'incertezze [...] a una terra di consapevolezza e di dialettica. (Consolo, 1997: 177-178)

Dei tre punti sopraelencati, solo il primo è notoriamente escluso, è di là da venire. Inoltre, questo movimento ad assetto triangolare, emanante dai brani d'apertura del capitolo, è illuminante riguardo alla poetica di Consolo e a come (e dove) egli si vede situato all'interno della tradizione letteraria siciliana: in una posizione intermedia tra quella occidentale e quella orientale, che è come dire, tra

3 Seguo le categorizzazioni e classificazioni genettiane della "transtestualità", vale a dire la trascendenza testuale del testo, rilevate da Gérard Genette (1997: 7-8): «si tratta appunto di quella che chiamerò d'ora in poi ipertestualità. Designo con questo termine ogni relazione che unisca un testo $\mathrm{B}$ (che chiamerò ipertesto) a un testo anteriore A (che chiamerò, naturalmente, ipotesto) sul quale esso si innesta in una maniera che non è quella del commento». 
storia e mito. I movimenti spaziali da est a ovest nel primo capitolo del romanzo, e la decisione dell'autore di abbracciare le tradizioni storiche occidentali degli scrittori dell'isola, si scorgono nello stesso incipit ${ }^{4}$. Nel libro-intervista Fuga dall'Etna, riferendosi proprio alla prima parte del romanzo, Consolo afferma:

Il libro è scritto nella prima parte in forma parodistica, mimetica, sarcastica se si vuole, quindi in negativo: faccio il verso a un erudito dell'Ottocento recluso nella sua mania antiquaria, che scrive i suoi saggi scientifici, che si occupa di malacologia, una materia quanto mai curiosa, eccentrica. (Consolo, 1993: 45)

Se gli elementi parodistici, mimetici e sarcastici sono davvero presenti, e in abbondanza, nella prima parte del romanzo, Consolo però non evidenzia tutti gli altri che danno "forma" a questo capitolo: un'angoscia delle influenze di altri scrittori, la ricerca di una nuova poetica, una concezione totalmente alterata di quello che è il genere del romanzo. L'analisi s'incentrerà proprio su questi elementi nascosti o impliciti, peraltro parzialmente rivelati dall'esame del graduale crescere del testo, gli inizi del quale si possono far risalire a un momento circostanziato della vita dell'autore, un evento che è l'emblema degli obiettivi dichiarati della sua poetica, vale a dire, la fusione dei due filoni, orientale e occidentale, della letteratura siciliana.

È mia ferma convinzione, che l'incontro organizzato da Consolo tra i suoi due mentori, il poeta Lucio Piccolo e Leonardo Sciascia, è il più chiaro esempio possibile del punto di partenza del futuro romanzo. Ne Le pietre di Pantalica, Consolo racconta l'evento tenutosi il 7 marzo 1965, una data degna di nota, perché coincidente con la prima messa officiata in lingua italiana dopo il Concilio Vaticano Secondo:

Sciascia arrivò da Caltanissetta al mio paese e assieme andammo da Piccolo. Al congedo, sulla porta, Piccolo solennemente disse allo scrittore, indicando con la mano su per le colline: «Sciascia, la invito a scrivere di queste nostre terre, di questi paesi medievali».

Avevo deciso di lasciare la Sicilia e di trasferirmi a Milano. «Non parta, non vada via» mi diceva Piccolo. "A Milano con tutti gli altri, rischia di annullarsi. La lontananza, l'isolamento danno più fascino suscitano interesse e curiosità.» Non potevo rispondergli che non ero ricco, che dovevo guadagnarmi la vita. Non potevo dirgli, soprattutto, che lì in Sicilia mi sembrava tutto finito, senza speranza, che a Milano, al Nord avevo la sensazione che tante cose si muovessero, che stesse per iniziare una nuova storia. (Consolo, 1988: 142-143 [Consolo, 2015: 599]) ${ }^{5}$

Ad un primo sguardo, nel brano sono proprio pochi gli accenni riferibili alla futura opera $\mathrm{Il}$ sorriso, perché Consolo taglia corto con la storia dell'incontro raccontando di altre esperienze con Piccolo. Invece nella Fuga dall'Etna riprende il racconto con un'interessante coda:

4 Per le discussioni sulla mappatura della letteratura siciliana del luogo, cfr. il mio saggio: O'Connell, (2005: 29-48); e O’Rawe (2007: 79-94).

5 La più antica traccia si trova in due quaderni autografi ( $M s 3, M s 4)$, per cui cfr. Messina (2009: 58 e n. 53). 
Questo uno dei ricordi più belli, che riprendo da Le pietre di Pantalica: «[...] "Sciascia, la invito a scrivere di queste nostre terre, di questi paesi medievali." "C’è qui Consolo," rispose Sciascia. "Consolo è ancora giovinetto," replicò Piccolo sarcasticamente (avevo trentatré anni!). Ma io presi quella frase come impegno verso Sciascia e come una sfida verso il barone.» Sciascia era rimasto affascinato da quel poeta, da quel gran personaggio che era Piccolo. Erano, i due scrittori, quanto di più diverso, di più lontano si potesse immaginare, eppure nutrivano, l'uno per l'altro, stima e ammirazione. (Consolo, 1993: 23-24) ${ }^{6}$

Non è da ritenere una coincidenza il fatto che siano esattamente queste nostre terre, quei paesi medievali a costituire il contenuto o retroscena del primo capitolo del futuro romanzo. La sfida sottaciuta di Consolo a Piccolo diventa poi col tempo anche sfida a tutta la letteratura siciliana, e il romanzo che ne risulta è un romanzo i cui antenati sono certo Verga, De Roberto, Pirandello, Vittorini e Tomasi di Lampedusa, ma i cui "istigatori" potrebbero considerarsi Piccolo e Sciascia: sia detto per inciso, richiamati rispettivamente dal protagonista Enrico Pirajno Barone di Mandralisca (Piccolo) e del deuteragonista Giovanni Interdonato (Sciascia).

Tredici anni di silenzio separano il primo romanzo di Consolo La ferita dell'aprile datato 1963 e Il sorriso. La concezione originale de Il sorriso, comunque, prende le mosse negli anni '60 ed è connessa, almeno dal punto di vista ideologico, al periodo di “acuta storia". Inoltre, è un romanzo siciliano in senso stretto, non solo perché tratta di storia siciliana, ma anche perché la sua nascita e il suo sviluppo sono databili al periodo 1963-1968, ovvero gli anni in cui Consolo visse in Sicilia prima di spostarsi a Milano definitivamente nel gennaio 1968. Ma Consolo, è chiaro, non smise di scrivere a metà anni '60 per riprendere la penna in mano soltanto a metà degli anni '70. Rimase attivo, sia sul piano creativo sia su quello giornalistico, durante gli anni che separano le date di pubblicazione dei due romanzi ${ }^{7}$. Tuttavia, ciò che si commenta è la preistoria effettiva del libro, ovvero come arrivò alla sua versione definitiva nel 1976. Quanto segue è la storia tracciata sulla fortuna di pubblicazione de Il sorriso dell'ignoto marinaio, dalle prime apparizioni fino ad oggi.

\section{Testo e testi: variazioni e varianti}

Nel numero luglio-settembre di «Nuovi Argomenti» del 1969, diretto da Alberto Carocci, Alberto Moravia e Pier Paolo Pasolini, appare per la prima

6 Le virgolette evidenzierebbero il passaggio citato da Le pietre di Pantalica fino a «una sfida verso il barone». In realtà, il passo da "C’è qui Consolo» a «una sfida verso il barone» non appaiono, come si è visto, in Le pietre di Pantalica.

7 Per la scrittura creativa, cfr. i racconti di quegli anni ora in La mia isola è Las Vegas (Consolo, 2012). Per quella giornalistica, anche se ne dà solo una visione parziale, Esercizi di cronaca (Consolo, 2013). Da non escludere sono anche gli articoli raccolti in Cosa loro. Mafie fra cronaca e riflessione. 1970-2010, (Consolo, 2017). Ed altri ancora, di vario argomento, risulta che ne siano conservati nell'Archivio Consolo. 
volta un racconto dal titolo Il sorriso dell'ignoto marinaio. A tutti gli effetti è ciò che diventerà il primo capitolo del futuro romanzo omonimo (Consolo, 1969: 161-174). Il racconto non presenta alcun antefatto o appendici. È di poco successivo, del 1975, un libro dallo stesso titolo con un'acquaforte di Renato Guttuso del famoso Ritratto d'uomo (edizione limitata in 150 copie). Il volume contiene i primi due capitoli di quello che sarà il romanzo che conosciamo (Consolo, 1975a). Corrado Stajano, amico di Consolo, avvalendosi di una celebre metafora pirandelliana, ha scritto un pezzo per Il Giorno per segnalare questa pubblicazione:

Adesso Manusè ha esaudito il gran sogno della vita, è diventato editore e c'è la possibilità, dicono gli uomini di penna, che questo libro che ha stampato, [...] possa creare un nuovo caso letterario. Perché qui si sono incontrate due corde pazze siciliane, quella di Manusè e quella dello scrittore del libro, o meglio dei primi due capitoli del libro pubblicato in questo volume, che gli editori, quando il romanzo sarà finito, certo si contenderanno, perché Il sorriso dell'ignoto marinaio è un nuovo Gattopardo, ma più sottile, più intenso del romanzo di Tomasi di Lampedusa, uno Sciascia poetico, di venosa lava sanguigna e insieme razionalmente freddo nei suoi teoremi dell'intelligenza. (Stajano, 1975)

La motivazione dell'articolo di Stajano sembra essere quella di voler forzare la mano di Consolo, spingendolo verso il completamento del romanzo. È anche notevole l'intento di fare pubblicità al romanzo futuro. Tuttavia, Stajano risulta anche molto ben informato del contenuto dei capitoli inediti e ciò suggerisce che al momento della pubblicazione della versione di Manusè la maggior parte del romanzo era già stata completata, o già comunque concettualmente concepita, e che Consolo ne stava discutendo apertamente con gli amici più fidati. Il romanzo completo, l'editio princeps, venne pubblicato l'anno successivo da Einaudi e fu accolto dagli elogi della critica (Consolo, 1976). Nel 1987 la Mondadori ne stampò un'edizione economica includendo una Introduzione firmata da Cesare Segre (Consolo, 1987). Nel 1991 Mursia pubblicò una seconda edizione aggiornata dell'antologia di Leonardo Sciascia e Salvatore Guglielmino Narratori di Sicilia, che accoglie, con annotazioni, la prima sezione del capitolo I de Il sorriso (Consolo, 1991). Nel 1992 Einaudi ripubblicò il romanzo (Consolo, 1992b) e nel 1995 ne apparve un'edizione scolastica, edita e annotata da Giovanni Tesio (Consolo, 1995).

Nel 1997 Mondadori ha pubblicato ancora una volta il romanzo insieme alla Nota dell'autore, vent'anni dopo (Consolo, 1997). Due successive riedizioni del romanzo sono state pubblicate sempre da Mondadori: la prima, nella collana Oscar scrittori del Novecento (Consolo, 2002); la seconda, in quella Oscar classici moderni (Consolo, 2004). Come si può intuire dalle date di pubblicazione, il periodo di gestazione de $\mathrm{Il}$ sorriso è stato decisamente lungo e in più va considerato che la versione originariamente pubblicata in «Nuovi Argomenti» differisce molto da quelle successive con una divisione in capitoli, anche perché parziale. 
Le varianti testuali tra le dieci versioni, edizioni e ristampe, databili dal 1969 al 1997, rivelano che Consolo ha rielaborato di continuo il suo testo. Che la maggior parte di esse siano quelle tra la versione di «Nuovi argomenti»e l'edizione del 1997, significa inoltre che Consolo ha ritoccato il testo dopo il $1976^{\circ}$. Le varianti si diversificano per tipologia e importanza, partono dai più banali errori di battitura per arrivare alle sostituzioni e alle rielaborazioni di interi paragrafi. Questo di per sé suggerisce che il capitolo I è una sorta di workin-progress, un testo sempre compulsato dall'autore e completato da una serie di aggiunte accorpate nel corso degli anni.

Lasciando da parte le fonti pubblicate, il cosiddetto emerso $o^{9}$, si può andare indietro fino al livello delle fonti precedenti alla pubblicazione e paratestuali che vanno a toccare il punto cruciale della genesi e gestazione del romanzo. Ho deciso di chiamarli pre-testi, nel senso che essi variano in tipologia, importanza e stato. Alcuni sono stati pubblicati e quindi dovrebbero rientrare nella categoria del paratesto, venendo così ad aumentare il nostro attuale bagaglio di nozioni sul romanzo.

\section{Pre-testi: genesi e gestazione}

Lo stato di incertezza che caratterizza i manoscritti autografi, i testi scritti a macchina, i saggi e gli altri "interventi autoriali", con particolare attenzione alla gestazione del testo, sono noti. Anche il problema di datare il materiale presenta non poche difficoltà. La tentazione, quando si ha a che fare con manoscritti e varianti testuali, è quella di sviluppare un approccio unilineare alla poetica dell'autore considerato; ascrivere a quell'autore certi presupposti fondamentali che tuttavia non sono facilmente verificabili. Nel caso di Consolo, al di là delle suddette edizioni del testo in forma di racconto e romanzo e dell'allettante realizzazione di varianti che intercorrono fra esse, esiste anche un ricco patrimonio di documenti non pubblicati. Nicolò Messina ha suggerito che sarebbe stato «illuminante, oltre che un'entusiasmante avventura, il poter penetrare grazie a un'edizione critica genetica all'interno della sua officina» (Messina, 1994: 40). Da allora, Messina si è imbarcato in questa avventura con una serie di articoli ispirati a un approccio filologico critico-genetico alle opere di Consolo, specialmente Il sorriso (Messina, 2005: 113-126). Oltre a ciò, Messina ha completato l'edizione critica del romanzo di cui si sentiva l'assoluta necessità e che è in se stessa un vero capolavoro di critica-genetica ${ }^{10}$. Poiché Messina ha lavorato sui manoscritti e dattiloscritti, non mi ci soffermerò.

8 L'edizione del 1997 è il testo tenuto come base per lo studio di tutti gli altri, essendo la versione che ha ricevuto l'ultimo ne varietur dell'autore. Concordo in questo con Messina (2005: 121-124).

9 Ricorro alla denominazione di Messina (2005: 117-121).

10 Messina (2009). L'approccio critico-genetico di Messina è basato sui seguenti studi: Hay (1979), Degala (1988), Gresillon (1994), Tavani (1996) e Contat e Ferrer (1998). 
Ai fini del nostro discorso, però, risulta assai significativo un dattiloscritto denominato $D s$, o piuttosto le pagine che l'accompagnano (Ds $2^{\circ}$ ), perché contengono una sorta di resoconto del futuro romanzo. In particolare, l'asserzione nella scheda $D s 2^{0}$ che «sono due capitoli di un romanzo (capitoli o racconti autonomi, perché, nelle intenzioni dell'autore, intercambiabili e combinatori come carte da giuoco)», pone una serie di questioni che sono rilevanti per la genesi e la gestazione del testo. Ci sono numerose sovrapposizioni, intrecci tematici e corrispondenze lessicali tra i capitoli I e II del romanzo. Dal punto di vista strutturale, la narrazione di entrambi i testi è in parte focalizzata attraverso Mandralisca (capitolo I) e Interdonato (capitolo II) con corrispondenze tra i due. Il fatto che i capitoli I e II siano intercambiabili e combinatori, postula un'influenza di Italo Calvino sul metodo strutturale di Consolo, in particolare del saggio Appunti sulla narrativa come processo combinatorio (Calvino, 1995: 199-219). Nella spiegazione di Calvino dell'ars combinatoria tanti elementi avrebbero potuto colpire Consolo, non ultimi le citazioni da Strutture topologiche nella letteratura moderna di Hans Magnus Enzensberger ${ }^{11}$, Calvino tra l'altro afferma anche:

La battaglia della letteratura è appunto uno sforzo per uscire fuori dai confini del linguaggio; è dall'orlo estremo del dicibile che essa si protende; è il richiamo di ciò che è fuori dal vocabolario che muove la letteratura. (1995: 211)

E che Calvino nel saggio ritorni sul simbolo del labirinto dopo l'analisi fattane nel precedente La sfida al labirinto (1962), non avrà lasciato indifferente Consolo tutto preso dalla sua curiosa, personale concezione siciliana del labirinto in $I l$ sorriso: cioè la chiocciola ${ }^{12}$.

La questione della genesi del romanzo si può far risalire ancora più indietro, all'attività giornalistica di Consolo da metà degli anni Sessanta in poi. Al riguardo ci si può anche chiedere quale influenza abbia potuto esercitare il giornalismo sui suoi sforzi creativi. Per tanti aspetti il giornalismo consoliano è come un'istantanea dei multiformi interessi nutriti in quel tempo: letterari, artistici, politici e civili; e quando tutto ciò viene visto attraverso la lente de Il sorriso, ci si apre un'intrigante entrée nel mondo della sua attività creativa e poetica ancora in via di sviluppo. Consolo ha scritto per Tempo illustrato e, durante il suo primo periodo a Milano, ebbe una regolare rubrica intitolata Fuori casa nel giornale palermitano $L^{\prime} O r a^{13}$. Tuttavia, il suo rapporto con $L$ 'Ora

11 Avrà una diretta influenza su Consolo, si sa, il saggio di Hans Magnus Enzensberger (1966: 7-22).

12 Calvino (1995: 99-117) dove (116) si sostiene: "Quel che la letteratura può fare è definire l'atteggiamento migliore per trovare la via d'uscita, anche se questa via d'uscita non sarà altro che passaggio da un labirinto all'altro. È la sfida al labirinto che vogliamo salvare, è una letteratura della sfida al labirinto che vogliamo enucleare e distinguere dalla letteratura della resa al labirinto». Consolo mette in scena la sua sfida al labirinto attraverso il personaggio secondario di Catena Carnevale in Il sorriso.

13 La rubrica, pubblicata dal dicembre 1968 al maggio 1969, è definita un «piccolo gioiello» da Nisticò (2001: 113); e si può rileggere in Consolo (2013: 179-223). 
precede il suo trasferimento a Milano ed è indissolubilmente legata con le sue attività in Sicilia ${ }^{14}$.

Nel 1965 e negli anni immediatamente adiacenti, un gruppo di intellettuali e scrittori cominciarono a frequentare il giornale, tra questi Sciascia, il fotografo Enzo Sellerio, lo scrittore Michele Perriera e Consolo stesso. Vittorio Nisticò, direttore de L'Ora nel periodo 1955-1975, ricorda con affetto la presenza e il contributo di Consolo in quegli anni ${ }^{15}$. Nel 1966, a seguito dell'omicidio per mano mafiosa del leader sindacale socialista Carmine Battaglia a Tusa (provincia di Messina), Consolo scrisse un breve racconto ispirato all'uccisione, Per un po' d'erba ai limiti del feudo, pubblicato ne L'Ora (16 aprile) un mese dopo l'evento $^{16}$. Anche se non può facilmente e strettamente rientrare nella categoria del giornalismo, il racconto, come sostiene Sciascia, è «una sorta di reportage giornalistico» (Consolo, 1967b: 429), ed è forse la prova più significativa della scrittura creativa di Consolo dopo La ferita dell'aprile, perché presenta in forma embrionale alcuni elementi linguistici, strutturali e tematici che saranno precipui de $I l$ sorriso.

Consolo, senza dubbio, ebbe come modello Le parole sono pietre di Carlo Levi, in particolare la terza sezione in cui si narra la sua visita a Francesca Serio, la madre del sindacalista socialista Salvatore Carnevale, assassinato anch'egli dalla mafia (Levi, [1955] 1979). Non è una coincidenza che Consolo abbia firmato in seguito l'introduzione per una nuova edizione del "libro-indagine", (Levi, 1979; Consolo, 1999a, 251-257) in cui scrive tra l'altro:

A Sciara, Levi ha trovato, sul filo sottile che inseguiva della nuova coscienza contadina, il punto più vero e più alto della realtà siciliana di quegli anni. E più vero e più alto si fa allora il tono del libro: le pagine su Francesca Serio di commozione rattenuta dal pudore, di parole scarne e risonanti. (Consolo, 1999a, 256)

Lo stesso senso di ingiustizia e indignazione permea Per un po' d'erba ai limiti del feudo, anche se nella storia di Consolo la figura della madre è abbattuta dal dolore e il compito di parlare viene lasciato alla figlia, «una giovane bellissima». All'inizio la incontriamo impegnata in un'attività che fa seriamente presagire le preoccupazioni testuali e metaforiche del futuro romanzo: «dietro i vetri di una piccola finestra, ricamava» (Consolo, 1967b: 432; Consolo, 2012: 20). Come personaggio, la figlia anticipa la figura liminale, altamente metaforica,

14 La prima collaborazione risalirebbe esattamente al 4 febbraio 1964, una recensione di Menabò, 6. Cfr. Salvatore Grassia, in Consolo (2013: 229-230).

15 Nisticò (2001: 113): «Amavamo di lui il garbo, la modestia, il senso di amicizia, gli accenni di sorridente ironia, non meno di quanto ci affascinassero i ricami della sua scrittura, la sua totale mediterraneità, quei fuochi improvvisi della sua passione letteraria e civile.» Il memoriale di Nisticò è senz'altro un affascinante resoconto di un giornale di sinistra in prima linea nella lotta contro la mafia in uno dei più difficili periodi storici.

16 Il racconto fu poi ripreso da Narratori di Sicilia, cit. [ed. 19671], (Consolo, 1967b: 429434); ora, col titolo Un filo d'erba al margine del feudo (Consolo, 2012: 18-22; 239). Sul caso Battaglia, cfr. Ovazza (1967) e Santino (2000: 232-233). 
della venticinquenne Catena del Sorriso, che nell'Antefatto il padre si augura di vedere "serena dietro il banco a ricamare» (Consolo, 1997: 11) ${ }^{17}$. Anche il cognome di Catena è intrigante: si chiama, infatti, Carnevale, nome che rafforza il legame con il "giovane", ventiduenne, Salvatore del racconto e con la famiglia di Le parole sono pietre. La presentazione della giovane ragazza da parte di Consolo suggerisce a Traina "sviluppi successivi della narrativa consoliana», come «l'insistenza sul dettaglio cromatico e sull'immagine "rubata" dal passante, che si fa quasi, emblematicamente, quadro o fotografia». (Traina, 2001: 16). Questa tecnica di sospensione della realtà tramite l'immagine fissa, una forma di ipotiposi, è un aspetto prevalente della narrativa più tarda di Consolo.

Il racconto di Consolo contiene inoltre una serie di espedienti e scelte lessicali che saranno ulteriormente ampliati nel romanzo. Ecco esempi di emergenza di scrittura "consoliana", quale poi impronterà il romanzo: «Il sole batteva [...] sulle pietre di via Murorotto e sul portale d'arenaria ricamata del Palazzo»; «[...] col suo castello sull'acqua smagliante e triangoli di vele sui merli» (Consolo, 1867b: 429 2 431; Consolo, 2012: 18-19). E successivamente, nel capitolo I de Il sorriso, leggiamo: «Vorticare di giorni e soli e acque, venti a raffiche, a spirali, muro d’arenaria che si sfalda [...]»; «Torrazzi d'arenaria e malta, ch' estollano i lor merli di cinque canne sugli scogli» (Consolo, 1997: 12; Consolo, 2015: 132). Inoltre, va sottolineato che la predilezione di Consolo per la catalogazione o inserzione, soprattutto di toponimi, è presente in maniera decisa nel racconto. Entrando nella città di Tusa, il narratore si mette a conversare con un «vecchio con lo scialle», che indica le montagne circostanti:

«Motta» [...]. E poi «Pettineo, Castelluzzo, Mistretta, San Mauro...» [...]. Stesi io il braccio nel vuoto oltre la ringhiera e indicai il mare. "Quelle macchie azzurre sono isole, Alicudi, Filicudi, Salina... Più in là c’è Napoli, il Continente, Roma...» «Roma» ripeté il vecchio. Volse le spalle al mare e continuò a indicare verso le montagne, ora con un breve cenno del capo: «Cozzo San Pietro, Cozzo Favara, Fulla, Foieri...». (Consolo, 1967b: 430; Consolo, 2012: 18)

Il nome di Roma non dice niente al «vecchio», egli ripete meccanicamente ed elenca rapidamente i toponimi del suo mondo, toponimi non registrati sulle mappe ufficiali, come se la loro enunciazione andasse a evocare una realtà diversa. Questo senso di "urgenza toponimica” e la sua sospensione sono ripetuti nel capitolo I del romanzo, dove ancora una volta l'elenco dei toponimi della costa tirrenica della Sicilia è predominante: «Erano del Calavà e Calanovella, del Lauro e Gioiosa, del Brolo...» (Consolo, 1997: 12; Consolo 2015: 128). L'antico passato greco di Tusa è evocato in una breve parentesi del racconto e anticipa il Consolo de Il sorriso:

La valle declinava dolce fino alla balza d'Alesa (le sue mura massicce, l'agorà, i cocci d'anfora e i rocchi di colonna affioranti tra gli ulivi, la bianca Demetra dal velo incollato sul ventre abbondante). (Consolo, 1967b, 431; Consolo, 2012: 19)

17 Il ricamo di Catena è una chiara metafora tessile del testo. Per ulteriori approfondimenti di questo aspetto consoliano, cfr. il mio saggio: O’Connell, (2003, 85-105). 
Il nome di Tusa deriva dall'arabo "Alesa al-tusah" (“Alesa la nuova”), quando l'antica città greca di Alesa perse il primato nella zona nella seconda metà del Ix secolo, e il centro fu spostato verso il sito dell'odierna Tusa (Ingrillì, 2000: 56). Nel romanzo, Consolo sancisce una sorta di nostalgia antico-archeologica tramite l'esposizione di ulteriori elenchi toponimici:

Erano Abacena e Agatirno, Alunzio e Calacte, Alesa... Città nelle quali il Mandralisca avrebbe raspato con le mani, ginocchioni, fosse stato certo di trovare un vaso, una lucerna o solo una moneta. (Consolo, 1997: 13; Consolo, 2015: 128129)

Oltre a manifestare le preoccupazioni più importanti del Mandralisca per l'archeologia e il suo rifiuto di affrontare la realtà contemporanea, nella fattispecie quel che si rivelerà essere un cavatore di pomice ammalato di silicosi, la frase si riferisce agli antichi toponimi greci della regione dei Nebrodi sulla costa tirrenica, alcuni dei quali erano già stati citati da Cicerone tra le città della Sicilia vittime di Verre:

Tyndaritanam, nobilissimam civitatem, Cephaloeditanam, Haluntinam [Alunzio], Apolloniensem, Enguinam, Capitinam perditas esse hac iniquitate decumarum intellegetis. (Verrine, II.3, 103)

Tuttavia nell'edizione del 1997 c'è una variante del testo: «Erano Abacena e Agatirno, Alunzio e Apollonia, Alesa...». Consolo ha chiaramente sostituito la diade Alunzio e Calacte con l'altra Alunzio e Apollonia con l'evidente intenzione di migliorare l'allitterazione della frase e ottenere un elenco alfabetico pressoché perfetto, e in tal modo ha rivelato che alcune rielaborazioni de facto hanno avuto luogo tra le due edizioni cronologicamente estreme dell'intero romanzo. Consolo suggerisce il motivo della sostituzione in un saggio celebrativo di Cefalù pubblicato nel 1999: "Nascere dov'erano soltanto echi d'antiche città scomparse, Alunzio Alesa Agatirno Apollonia, che con la loro iniziale in A facevano pensare agli inizi della civiltà»(Consolo, 1999b: 17). Le somiglianze sono evidenti e ci permettono di visualizzare i processi che hanno delineato la creazione di questo capitolo. Non è un caso, quindi, che questa invocazione alla lettera A, alla storia antica, alla storia seppellita sotto i toponimi odierni dovesse avvenire nella fase iniziale di un romanzo intimamente connesso tanto con ciò che la storia nasconde quanto con ciò che essa rivela. Apollonia, inoltre, si ritiene che fosse l'antico toponimo dell'odierna San Fratello, un paese che ha una funzione estremamente metaforica in gran parte delle opere di Consolo, non ultima $\mathrm{Il} \mathrm{sorriso}^{18}$. Consolo sta, quindi, mettendo uno dei suoi marker, o

18 La città è evocata, in vario grado, in La ferita dell'aprile (1963), Lunaria (1985) e nel racconto «I linguaggi del bosco», in Le pietre di Pantalica (1988). In particolare il sanfratellano, usato o semplicemente citato da Consolo a più riprese, sembra attirare l'attenzione dello scrittore per la natura e ricchezza di lingua "periferica", voce di una cultura particolare in netto contrasto con la lingua omologata "centrale" cui ricorre la cultura dominante. 
segnali, alludendo a qualcosa che acquisisce via via importanza con il procedere del racconto.

L'evocazione di antichi toponimi era riapparsa invero in Le pietre di Pantalica, in quel Il barone magico che si avvale ancora una volta della toponomastica poetica e suggerisce con gli stessi toponimi (e qualcuno nuovo) non solamente una topografia autoriale personalizzata, ma anche la loro contiguità con l'atto di scrittura e gli inizi della parola:

Qui era un tempo la città antica d'Agatirno, una delle città lungo questa costa che, coi loro nomi comincianti in A (Abacena, Alunzio, Apollonia, Amestrata, Alesa...) fanno pensare ai primordi, alle origini della civiltà. (Consolo, 1988: 147; Consolo, 2015: 603)

Il passo tratto da Il sorriso condivide anche somiglianze con un'altra opera d'impostazione storica parallela, I vecchi e i giovani di Pirandello:

Via Atenea, Rupe Atenea, Empedocle... - nomi: luci di nomi, che rendeva più triste la miseria e la bruttezza delle cose e dei luoghi. L’Akragas dei Greci, l'Agrigentum dei Romani, eran finiti nella Kerkent dei Musulmani, e il marchio degli Arabi era rimasto indelebile negli anni e nei costumi della gente. (Pirandello, 1973: 163)

Il suo stato di intertesto è ulteriormente rafforzato appena più avanti, nello stesso quarto paragrafo, quando Consolo sostiene: «Ma quelle, in vero, non sono ormai che nomi, sommamente vaghi, suoni, sogni» (Consolo, 1997: 13; Consolo, 2015: 129). Le affinità tra il pirandelliano nomi: luci di nomi e il consoliano nomi [...], suoni, sogni sono degne di nota.

Forse sarà più significativo che Per un po' d'erba ai limiti del feudo anticipi la tendenza di Consolo a usare documenti storici come discorsi compensativi all'interno delle proprie narrazioni, una pratica più pienamente realizzata ne $I l$ sorriso dell'ignoto marinaio ${ }^{19}$.

Sul portone del municipio era scolpito lo stemma della città: un grosso cane muscoloso sopra una torre, le zampe posteriori contratte, sul punto d'avventarsi, i denti scoperti. (1860: «In più luoghi, come a Bronte, a Tusa e altrove, i Consigli municipali, costituiti dai Governatori distrettuali, erano composti di elementi della grossa borghesia o dell'aristocrazia di proprietari terrieri, avversi alle rivendicazioni contadine e ai fautori e capi del movimento per la divisione delle terre demaniali»). (Consolo, 1967b: 432; Consolo, 2012: 20)

La parte del passo in corsivo è tratta da un documento contemporaneo allo sbarco di Garibaldi in Sicilia e sottolinea un fondamentale fattore storico del Risorgimento in Sicilia: l'opposizione delle classi dominanti in Sicilia al decreto del 2 giugno 1860 «col quale Garibaldi ordinava la divisione delle terre demaniali mediante sorteggio a tutti i capi di famiglia sprovvisti di terra, riservando una quota certa ai combattenti della guerra di liberazione ed ai loro eredi» (Romano, 1952: 139).

19 Segre esamina la funzione narrativa di tale pratica consoliana, anche se l'attenzione del saggio è incentrata esclusivamente sui documenti storici riprodotti in appendice e non su quelli incorporati nel testo stesso (Segre, 2005: 129-138). 
L'amara ironia che scaturisce dall'inclusione del documento sottolinea il fatto che in 106 anni poco era cambiato nella vita e nelle aspirazioni dei diseredati. E, forse, per questa ragione che Sciascia nella storia scorge il «gioco gattopardesco delle forze della conservazione» (Consolo, 1967b: 429). Da parte sua, di fronte alla staticità del corso degli eventi, Onofri sottolinea che l'immobilità storica, sancita dal documento legislativo, "trova finale suggello nella letteratura, testimonianza abbastanza precoce di quello che possiamo definire il circolo ermeneutico consoliano» (Onofri, 1995: 232). Tuttavia, a permeare queste pagine è soprattutto l'impegno di Consolo a favore della giustizia sociale. Nel racconto, dunque, si possono ritrovare le tracce evidenti del suo primo tentativo di scrivere qualcosa sui paesi medievali additati da Piccolo, però attraverso il filtro dell'impegno di matrice sciasciana, cioè della sua stessa volontà di abbracciare i temi della giustizia sociale e politica.

Sulla questione dell'inserimento di documenti storici nel narrato, il primo capitolo del Sorriso offre un accattivante esempio del metodo di Consolo. È riscontrabile nel diciannovesimo paragrafo e dà nell'occhio proprio per quel che rivela delle procedure narrative impiegate dallo scrittore nei suoi testi multiformi. Il paragrafo, presentato per la sua maggior parte in corsivo, suggerisce una citazione o, almeno, una condizione narrante alternativa, una voce o un punto di vista diversi. Nel romanzo, l'unico altro luogo in cui una vasta porzione di testo è riportata in corsivo è nel Capitolo $\mathrm{v}, \mathrm{Il}$ Vespero, in cui il passo incastonato non in tondo è mutuato direttamente da I promessi Sposi di Alessandro Manzoni: è la descrizione del momento della conversione dell'Innominato piegata da Consolo a tratteggiare il "tempo" e le sensazioni del personaggio Peppe Sirna (Consolo, 1997: 106; Consolo, 2015: 204-205; Manzoni, 2002: 409). Tranne il corsivo non c'è nel testo nessuna indicazione che si tratti di una citazione manzoniana.

Nessuna indicazione, né note a pie' di pagina né richiami all'autore, soccorre il lettore, il quale non può far altro che accettare la stranezza del passo ed andare avanti con la narrazione. Il brano è interrotto dall'improvviso cambiamento di registro impresso da Enrico Pirajno, barone di Mandralisca, che esclama: «Uh, ah, cazzo, le bellezze!» (Consolo, 1997: 20; Consolo, 2015, 134), poiché la sua immaginazione prende il sopravvento sul resto del paragrafo. Ciò che precedeva in corsivo, però, non è un parto dei rimuginii del protagonista del Sorriso, ma un qualcosa di abbastanza strano ed estraneo, è il frutto del pensare altrui, benché sia la mente del barone Mandralisca a rievocarlo. Si ha un indizio nella frase finale del paragrafo:

Avrebbe fottuto il Bìscari, l'Asmundo Zappalà, l'Alessi canonico, magari il cardinale, il Pèpoli, il Bellomo e forse il Landolina.

Questo forse il Landolina è l'unica allusione, nel testo, ad una possibile fonte, anche se il lettore medio non ha modo né è in grado di saperlo. La parte in corsivo del brano, cioè i tre quinti del paragrafo, è, in effetti, una citazione diretta da una fonte che non sarebbe stata familiare per il Mandralisca, sebbene Consolo la abbia consultata come campione di stile di prosa degli intellettuali siciliani del tardo 
Settecento e del primo Ottocento. La fonte è proprio il Cavalier Saverio Landolina (1753-1814), figura dominante dell'archeologia siciliana all'inizio del xIX secolo. La sua fama e la sua posizione di rilievo si dovevano alla scoperta della famosa Venere Anadiomene, fatta nel 1803 (Dizionario dei Siciliani illustri, 1939). Il testo in questione è da ricercare in una lettera datata 29 gennaio 1807 e indirizzata all'allora «Sopraintendente generale alle antichità», un certo Soratti (Agnello, 1972: 218-219; Consolo, 1997: 19-20; Consolo, 2015: 134):

Passando a visitare li monumenti del Tindaro ebbi il dispiacere di non ritrovare il più bel pezzo, che l'altra volta vi avevo ammirato. Erano due piedi con le gambe fino alle cosce di un giovane ignudo di elegantissimo greco lavoro, con un'ara dal lato sinistro ben ornata, di marmo alabastro bianco. Osservai ancora due grossi pezzi di marmo statuario, che insieme formavano il busto di un uomo di statura gigantesca; in uno dei detti pezzi si vede la corazza ornata di bassi rilievi, tra $i$ quali si distinguano una bulla pendente sul petto con una testa molto crinita come si osserva in molte nostre medaglie. Dalla spalla destra era pendente sopra la mammella una fettuccia lavorata. Su la spalla sinistra era elegantemente rilevato il gruppo del pallio che doveva coprire le spalle. Sopra il ventre erano due ippogrifi. L'altro pezzo di marmo era il rimanente della corazza, cioè le fibule e le bulle pendenti sopra il sago che copriva le cosce le quali si vedono tagliate. Le bulle erano tutte figurate con varie teste di animali e qualcuna umana. L'esistenza di questi pezzi nel Tindaro mi fa sospettare che potevano appartenere ad una statua dei Dioscuri, descritti sempre dai poeti in abito militare.

Il brano di Landolina è citato pressoché alla lettera e le sole inter/estrapolazioni eseguite da Consolo vanno individuate nei tempi verbali e in quei segmenti del brano che si riferiscono al Landolina stesso. Questo tipo di intertestualità è abbastanza sconcertante per il lettore e, una volta rilevato, mostra il modo in cui si forgia lo stile di Consolo: testi dentro testi, siano essi citazioni poetiche di scrittori canonici o citazioni dirette da oscuri testi archeologici del XIX secolo. La citazione letteraria diretta si può considerare ammissibile, se accettiamo che il punto di vista narrativo è qui quello del Mandralisca, com'è peraltro accertabile nel resto di questo capitolo iniziale, ma la citazione diretta da lettere di argomento archeologico è più problematica.

Segre scrive che Consolo condivide con Gadda «la voracità linguistica, la capacità di organizzare un'orchestra di voci, il risultato espressionistico» e, assecondando le riflessioni di Bachtin, aggiunge che il plurilinguismo di Consolo è anche «nettamente plurivocità» (Segre, 1991: 83-85). Al riguardo i commenti bachtiniani sull'enciclopedismo nel genere del romanzo sono rilevanti, specialmente in quelli da lui definiti romanzi della seconda linea. Questo tipo di romanzo tende all'enciclopedicità dei generi, e si avvale anche dei generi inseriti. Il fine principale è introdurre nel romanzo la pluridiscorsività, la varietà delle lingue di un'epoca. Scrive Bachtin che i

generi extraletterari sono introdotti non per "nobilitarli" e "letteraturizzarli" ma proprio perché sono extraletterari, perché era possibile introdurre nel romanzo una lingua extraletteraria (persino un dialetto). La molteplicità delle lingue dell'epoca deve essere rappresentata nel romanzo (Bachtin, 2001: 218). 
L'uso sapiente di Consolo di mescolare generi letterari ed extraletterari contrassegna Il sorriso come un complesso romanzo polifonico. Tuttavia, la funzione di memoria qui è profondamente testuale e quindi comparabile a un palinsesto.

Un altro brano altamente significativo di Consolo in questi anni Sessanta appare ne L'Ora ed è un pezzo dedicato a Lucio Piccolo. L'articolo intitolato Il barone magico celebra in apparenza l'impresa poetica di Piccolo (Consolo, $1967 a)^{20}$. Il testo di Consolo sarebbe un pretesto per la presentazione di tre poesie inedite di Piccolo e un frammento della sua prosa Balletto in tre tempi: L'esequie della luna ${ }^{21}$. Come nel caso di Per un po' d'erba ai limiti del feudo, Il barone magico anticipa molto del futuro Sorriso, ma se l'attenzione nel racconto era imperniata sull'indignazione civile dell'autore, il pezzo su Piccolo vede un Consolo lontano dalle questioni politiche e, invece, fortemente immerso nelle potenzialità magiche della parola, radicate nella seduzione del testo poetico. Se il primo era sciasciano nei presupposti e risultati, il secondo è, in gran parte, piccoliano nella sua espressività. Queste tendenze conflittuali dovevano risolversi nell'intensa fusione della scrittura de Il sorriso, in cui Consolo stesso divenne, per Stajano, uno "Sciascia poetico» (Stajano, 1975). E - si potrebbe forse aggiungere - anche un "Piccolo impegnato".

Questi, poi, sono gli scritti di Consolo fino al momento del trasferimento a Milano nel 1968, scritti che rivelano chiaramente che egli aveva già in mente le coordinate del futuro romanzo. Non che il suo rapporto con il giornale palermitano si fosse concluso con la decisione di lasciare la Sicilia, anzi le collaborazioni di Consolo con L'Ora s'intensificarono e divennero più "tradizionalmente" giornalistiche. I temi affrontati per il quotidiano erano abbastanza diversificati e variavano dalla politica, alla cronaca, alla critica d'arte, alle interviste e recensioni, come anche ad alcuni saggi di produzione creativa. Nel 1975 Consolo tornò in Sicilia a lavorare al romanzo e ricominciò a frequentare L'Ora. La testimonianza di Nisticò è interessante per l'attenzione prestata agli interessi poliedrici di Consolo e alle attività da lui svolte contemporaneamente alla scrittura de $I l$ sorriso:

Nei primi mesi del '75 Consolo si trasferì per un po' di tempo a Palermo. [...] si buttò con manifesta gioia in un intenso lavoro giornalistico. [...] Insomma, un bel bagno mediterraneo di umile giornalismo, mentre tra un servizio e l'altro trovava il luogo e il silenzio dove ripararsi per dare gli ultimi ritocchi a Il sorriso dell'ignoto marinaio: il capolavoro che da lì a qualche mese lo avrebbe consacrato tra gli eredi della grande letteratura che la Sicilia ha dato alla nazione. A dicembre ne pubblicammo in anteprima un capitolo: la festa in casa del barone Mandralisca. (Nisticò 2001: 113-114)

20 La maggior parte del materiale dell'articolo costituisce la prima sezione del suo successivo «Il barone magico» (Consolo, 1988: 133-135).

21 Le poesie erano Plumelia, L'andito e quella che sarà poi intitolata Non fu come credest $i$ per lo scatto. 
Un altro esercizio giornalistico con attinenza diretta alla formazione del capitolo I è un articolo, datato al 1970, fremente d'indignazione civile per le deplorevoli condizioni di lavoro dei cavatori di pomice dell'isola di Lipari. Originariamente intitolato «Il paese dei vivi pietrificati», fu pubblicato su Tempo illustrato, ma sotto altro titolo e solo dopo aver subito pesanti interventi modificatori (Consolo, 1970) ${ }^{22}$. Ancora una volta, affiora il primo capitolo del romanzo che si occupa del destino dei cavatori di pomice di Lipari e della strana malattia da cui sono affetti: la silicosi, popolarmente conosciuta come Male di pietra. L'articolo di Consolo è degno di nota, in quanto mette in risalto un misto di strategie del discorso significative anche per la sua poetica narrativa (Consolo, 1997: 177). Le preoccupazioni di Consolo sono molteplici, ma ciò che dà forza all'articolo è nel fondo la messa a fuoco della percentuale insolitamente alta a Lipari di malati di silicosi e della loro breve aspettativa di vita. Come chiarisce l'ignoto marinaio del romanzo, a provocare la malattia è l'estrazione della pietra pomice senza rispettare le più elementari misure di sicurezza. Consolo definisce le cave «un kafkiano teatro di vita penale dove si aspetta da sempre il messaggio dell'imperatore» ${ }^{23}$. È una sorta di altra controversia liparitana, ma diversa da quella della Recitazione sciasciana, in quanto lo sfondo non è settecentesco e Consolo vi delinea una storia dei cavatori di pietra pomice sull'isola a partire dal 1838, quando il monopolio è dato in appalto ad un francese di nome Gabriel Barthe. Lo scrittore si addentra nella battaglia giudiziaria tra questi e il vescovo di Lipari Giovampietro Natoli per il controllo delle miniere: il vescovo ottenne la proprietà delle terre, rifacendosi a un decreto del re Roberto I d'Altavilla datato $1084^{24}$ ! E non mancano accenni alla moglie del Guiscardo ovvero Adelasia di Monferrato, evocata nel primo capitolo del romanzo e sepolta a Patti. Questa serie di informazioni a prima vista insignificanti permette a Consolo di presentare l'effettivo proprietario delle cave di pietra pomice al momento della redazione dell'articolo: la mafia, legata al finanziere Michele Sindona, nato a Patti $^{25}$. Tuttavia, che le condizioni di lavoro dei cavatori non fossero cambiate

22 Sono grato a Caterina Consolo per avermi fornito una copia dell'originale Il paese dei vivi pietrificati, composto da 5 cartelle battute a macchina con annotazioni e correzioni di mano dell'autore, e aggiunte vergate da Caterina Consolo: il toponimo «Lipari» (indicante il tema), la data di redazione: «settembre», e il futuro del testo: «[pubblicato non integralmente]». La cartella finale è datata: «3 settembre 1970».

23 «Il paese dei vivi pietrificati», cit., c.3. Omesso nella versione apparsa in Tempo illustrato.

24 Consolo cita dal documento storico riportandolo ne «Il paese dei vivi pietrificati», ma il passaggio non compare in "Così la pomice si mangia Lipari».

25 Il banchiere Sindona fu una figura di spicco che ebbe contatti stretti con la mafia, leader politici italiani e la Loggia Massonica di Licio Gelli: la P2 (ben nota per gli scandali in cui fu coinvolta). Cfr. Renda (1998: 400-404); Lupo (1996: 262-271). L'editore di Tempo Illustrato soppresse evidentemente ogni riferimento a Sindona e al fatto che il manager dell'impianto Italpomice, Gebhart Raisch, era presumibilmente un ex Maggiore delle SS. 
dal 1852, anno dell'azione del primo capitolo de $\mathrm{Il}$ sorriso, è ben descritto nel pezzo che segue:

Alle spalle di Canneto è il monte Pelato, il monte grigio-bianco con pomice con tra le gole radi cespugli verdastri. [...] Gli operai sono dentro le gallerie sparsi qua e là per il costone. Con solo le mutande addosso, sotto questo sole di agosto, sono neri, piccoli e neri contro il bianco abbagliante. Sembrano ragni o scarafaggi che si muovono sopra una parete di calce o di sale.

Dall'altra parte della strada vi è il burrone che precipita fino al mare. Dalla costa si partono e vanno fino al largo snelli pontili neri, geometrici, sui quali scorrono $i$ nastri trasportatori che riempiono le stive delle navi attraccate all'altro capo ${ }^{26}$.

Il passo ha notevoli affinità con un altro del capitolo I de $\mathrm{Il}$ sorriso in cui Mandralisca svia il minuzioso esame di Interdonato permettendo alla sua immaginazione di proteggerlo dalla vista di uno di questi 'cavatori' e rivelando così che Consolo sta visualizzando la scena attraverso il filtro del suo testo:

Al di là di Canneto, verso il ponente, s'erge dal mare un monte bianco, abbagliante che chiamasi Pelato. Quivi copiosa schiera d'uomini, brulichìo nero di tarantole e scarafaggi, sotto un sole di foco che pare di Marocco, gratta la pietra porosa col piccone; curva sotto le ceste esce da buche, da grotte, gallerie; scivola sopra pontili esili di tavole che s'allungano nel mare fino ai velieri. Sotto queste immagini il Mandralisca cercava di nascondere, di rimandare indietro altre che in quel momento (frecce di volatili nel cielo di tempesta migranti verso l'Africa, verdi chiocciole segnanti sulla pietra strie d'argento, alte flessuose palme schiudenti le vulve delle spate con le bianche pasquali inflorescenze... $)^{27}$

Il paese dei vivi pietrificati è poi un esempio di come i processi creativi di Consolo abbiano chiaramente influenzato la sua scrittura giornalistica, in un'inversione di tendenza rispetto agli scritti per L'Ora fin qui considerati. Questi articoli dimostrano che Consolo stava riconsiderando i temi Risorgimentali da tanti punti di vista e in differenti prospettive.

Un ultimo punto da trattare, attinente alla gestazione del romanzo, è quello degli scritti consoliani relativi, ovvero ispirati, all'arte figurativa. Questa scrittura differisce considerevolmente da quella della produzione giornalistica ed è fatta principalmente di presentazioni per mostre di artisti contemporanei. Una in particolare, manifestando l'innata vocazione di Consolo a usare forme metriche nella prosa, esercitò una diretta influenza sulla gestazione del capitolo I. Il testo, intitolato Marina a Tindari, fu scritto per la mostra personale di Michele Spadaro tenutasi a Como presso la Galleria Giovio il 15-30 aprile 1972. Un centinaio di copie del testo della presentazione di Consolo venne pubblicato

26 «Il paese dei vivi pietrificati», cc. 2-3. La parte di testo in corsivo è stata omessa nell'articolo di Tempo illustrato.

27 Consolo (1969) e Consolo (1975a) presentano entrambi la variante precedente «sotto un sole di foco che pare di Morèa». Consolo ha scritto un altro pezzo per Tempo illustrato (2 ottobre 1971) con il titolo "C'era Mussolini e il diavolo si fermò a Cefalù». L'articolo porta avanti un'indagine sulla residenza di Aleister Crowley a Cefalù nei primi anni venti del Novecento ed è il primo indizio del futuro Nottetempo, casa per casa (1992). 
nello stesso anno dal fratello dell'artista, Sergio Spadaro, insieme a un breve saggio (Consolo, 1972) ${ }^{28}$. In Marina a Tindari, dopo un'introduzione di prosa prelevata direttamente da Consolo (1969), seguono ventiquattro versi:

Quindi Adelasia, regina d'alabastro,

ferme le trine sullo sbuffo,

impassibile attese che il convento si sfacesse.

- Chi è, in nome di Dio? - di solitaria

badessa centenaria in clausura

domanda che si perde per le celle,

i vani enormi, gli anditi vacanti.

- Vi manda l'arcivescovo? -

E fuori era il vuoto.

Vorticare di giorni e soli e acque,

venti a raffi che, a spirali, muro

d'arenaria che si sfalda, duna

che si spiana, collina,

scivolìo di pietra, consumo.

Il cardo emerge, si torce,

offre all'estremo il fiore tremulo,

diafano per l'occhio cavo

dell'asino bianco.

Luce che brucia, morde, divora

lati spigoli contorni,

stempera toni macchie, scolora.

Impasta cespi, sbianca le ramaglie,

oltre la piana mobile di scaglie

orizzonti vanifica, rimescola le masse. (Consolo, 1972: 15-16)

Nel primo capitolo di Consolo (1975a) il paragrafo 14 è un chiaro esempio delle numerose aggiunte testuali di questa edizione. Il paragrafo è costituito proprio da questo testo in versi di Marina a Tindari, ricondotto, per così dire, all'originaria prosa del catalogo, e in questa forma interpolato tale e quale da Consolo in nell'edizione di $1975 \mathrm{a}^{29}$. Echi di T.S. Eliot, Salvatore Quasimodo e Piccolo sono evidenti sin da una lettura iniziale. Il dato significativo, però, è che la poetica di Consolo comporti anche l'innesto di altri testi nel romanzo. Questo accorpamento non è solo una forma di autocitazione, ma un radicale spostamento di materiali testuali e potenzialità poetiche: cioè la nuova poetica

28 Consolo scrisse anche per la personale di un altro pittore: Luciano Gussoni, Villa Reale di Monza, 10-30 novembre 1971. Il titolo della presentazione era, nota interessante, Nottetempo, casa per casa. Secondo Messina (2005: 123), il testo scritto per la mostra avrebbe avuto una diretta ripercussione nella costruzione del capitolo viI de Il sorriso, dove sarebbe stato rifuso. Cfr. Messina (2009: 390-393); e inoltre p. 592-621 e 623-627, con le anastatiche del catalogo di Spadaro e di Marina a Tindari, seguite da quella del catalogo di Gussoni.

29 La disposizione in versi è opera del curatore del libretto, Sergio Spadaro, che ha inteso così visualizzare i metri intravisti nella prosa consoliana per il catalogo originario. Per un'analisi delle forme metriche nella prosa di Consolo, cfr. Finzi e Finzi (1978: 121-135). 
di Consolo comporta l'accumulo di diversi testi di varia provenienza, siano essi giornalistici, creativi o di ambito saggistico, in uno spazio a metà fra il polifonico e il palinsesto di singolare gestazione autoriale. Inoltre, in questo particolare esempio, lo spostamento di materiali testuali investe il rapporto di Consolo con l'arte figurativa e le potenzialità proteiformi di parola e immagine. Altrove lo scrittore ha dichiarato:

[...] io non ho mai scritto una recensione di tipo logico critico dei pittori. I pittori mi interessavano quando mi davano lo spunto per scrivere delle pagine di tipo lirico narrativo, ed allora poi utilizzavo queste presentazioni per scrivere quelli che io chiamo gli 'a parte', la parte del coro quando s'interrompe la narrazione. Queste digressioni di tipo lirico espressivo che i latini chiamavano 'cantica'. (Consolo, 2006: 235)

Questo tipo di poetici a parte o di elementi lirico-narrativi a carattere digressivo, sono presenti con maggior frequenza nell'opera più tarda di Consolo e vi assolvono una funzione decisamente corale. L'esempio dedotto dal capitolo i de Il sorriso rappresenta la prima apparizione di questa procedura unica nell'opera di Consolo, una procedura che suggerisce anche il suo ruolo epifanico all'interno della prosa. Non si tratta di un'ekphrasis nel senso stretto del termine, ma la fonte prima svolge una funzione ecfrastica nel suo accentuare l'articolazione del campo visivo.

Così, queste fonti testuali, giornalistiche, saggistiche e creative, insieme alle varianti testuali, offrono un aiuto inestimabile per la ricostruzione del capitolo I de Il sorriso dell'ignoto marinaio. Esse evidenziano i momenti salienti della gestazione e forniscono spunti di approfondimento delle preoccupazioni dell'autore; sono parte integrante dei processi creativi del romanzo e ampliano la nostra conoscenza degli aspetti oscuri dell'intero testo; soprattutto, ci permettono di vedere Il sorriso come un prodotto testuale degli anni attorno al 1960, periodo intimamente collegato agli anni di Consolo in Sicilia. Una volta a Milano, lo scrittore iniziò l'arduo cammino di tracciare la forma del suo romanzo, di riunirvi tutti i disparati elementi di questo testo mutevole.

\section{Bibliografia}

AA.VV., 1939, Dizionario dei Siciliani illustri, ristampa anastatica, Palermo, F. Ciuni Libraio Editore.

Agnello G. (a cura di), 1972, «Le antichità di Tindari nel carteggio inedito di Saverio e Mario Landolina», Estratto dall'Archivio Storico Siciliano Serie III Vol. xx, Palermo, La Società Siciliana per la Storia Patria, p. 218-219.

Bachtin M., 2001, Estetica e romanzo, trad. Clara Strada Janovič, Torino, Einaudi. Calvino I., 1995, Una pietra sopra, Milano, Mondadori.

Consolo V., 1967a, «Il barone magico: Lucio Piccolo», L'Ora, 17 febbraio.

Consolo V., 1967b, «Per un po' d'erba ai limiti del feudo», in: Narratori di Sicilia, a cura di Leonardo Sciascia \& Salvatore Guglielmino, Milano, Mursia, p. 429-434. 
Consolo V., 1969, «Il sorriso dell'ignoto marinaio», Nuovi Argomenti, 15 (lugliosettembre), p. 161-174.

Consolo V., 1970, «Così la pomice si mangia Lipari», Tempo illustrato, 17 ottobre.

Consolo V., 1971, «C’era Mussolini e il diavolo si fermò a Cefalù», Tempo illustrato, 2 ottobre.

Consolo V., 1972, Marina a Tindari, a cura di Sergio Spadaro, Vercelli, Arti grafiche Cav. Piero De Marchi.

Consolo V., 1975a, Il sorriso dell'ignoto marinaio, Milano, Gaetano Manusè.

Consolo V., 1975b, «Un suggestivo anticipo del nuovo romanzo di Vincenzo Consolo. Festa in casa del barone Mandralisca», L'Ora, 9 dicembre.

Consolo V., 1976, Il sorriso dell'ignoto marinaio, Torino, Einaudi.

Consolo V., 1985, Lunaria, Torino, Einaudi.

Consolo V., 1987, Il sorriso dell'ignoto marinaio, Milano, Mondadori.

Consolo V., 1988, Le pietre di Pantalica, Milano, Mondadori.

Consolo V., 1991, «Il sorriso dell'ignoto marinaio», Narratori di Sicilia, a cura di Leonardo Sciascia e Salvatore Guglielmino, Milano, Mursia, p. 387-395.

Consolo V., 1992a, Nottetempo, casa per casa, Milano, Mondadori.

Consolo V., 1992b, Il sorriso dell'ignoto marinaio, Torino, Einaudi.

Consolo V., 1993, Fuga dall'Etna. La Sicila e Milano, la memoria e la storia, Roma, Donzelli.

Consolo V., 1994, L'olivo e l'olivastro, Milano, Mondadori.

Consolo V., 1995, Il sorriso dell'ignoto marinaio, a cura di Giovanni Tesio, Milano, Elemond Scuola.

Consolo V., 1997, Il sorriso dell'ignoto marinaio, Milano, Mondadori.

Consolo V., 1999a, Di qua dal faro, Milano, Mondadori.

Consolo V., 1999b, «La scoperta di Cefalù (come un racconto)», in: Vincenzo Consolo, Giuseppe Leone, Cefalù, Palermo, Bruno Leopardi.

Consolo V., 2002, Il sorriso dell'ignoto marinaio, Milano, Mondadori.

Consolo V., 2004, Il sorriso dell'ignoto marinaio, Milano, Mondadori.

Consolo V., 2006, «Clausura de las jornadas», Lunaria vent'anni dopo, a cura di Irene Romera Pintor, Valencia, Generalitat Valenciana, p. 235-237.

Consolo V., 2012, La mia isola è Las Vegas, Milano, Mondadori.

Consolo V., 2013, Esercizi di cronaca, Palermo, Sellerio.

Consolo V., 2015, L'opera completa, a cura e con un saggio introduttivo di Gianni Turchetta e uno scrtto di Cesare Serge, Milano, Mondadori, «I Meridiani».

Consolo V., 2017, Cosa loro. Mafie fra cronaca e riflessione. 1970-2010, Milano, Bompiani.

Contat M., Ferrer, D. (a cura di), 1998, Pourquoi la critique génétique? Méthodes, théories, Paris, CNRS Éditions.

Contini G., 1970, Varianti e altra linguistica. Una raccolta di saggi (1938-1968), Torino, Einaudi.

Enzensberger H. M., 1966, «Letteratura come storiografia», Il menabò, 9, p. 7-22. 
Finzi A., Finzi, M., 1978, «Strutture metriche della prosa di Consolo», Linguistica e letteratura, 3: 2, p. 121-135

Genette G., 1997, Palinsesti: la letteratura al secondo grado, Torino, Einaudi.

Gresillon A, 1994, Éléments de critique génétique. Lire les manuscrits modernes, Paris, P.U.F.

Hay L. (a cura di), 1979, Essais de critique génétique, Paris, Flammarion.

Ingrillì F., 2000, Paesi e paesaggi dei Nebrodi, Brolo, Ermes dei Parchi.

Levi C., [1955] 1979, Le parole sono pietre, Torino, Einaudi.

Lupo S., 1996, Storia della mafia: dalle origini ai nostri giorni, Roma, Donzelli.

Manzoni A., 2002, I promessi sposi (1840), a cura di Salvatore S. Nigro, Milano, Mondadori.

Messina N., 1994, «Due contributi alla lettura di Vincenzo Consolo tra ecdotica e "Quellenforschung"», Cuadernos de Filología Italiana, 1, 39-46

Messina N., 2005, «Per una storia di "Il sorriso dell'ignoto marinaio" di Vincenzo Consolo», Quaderns d'Italià, 10, p. 113-126

Messina N., 2009, Per un'edizione critico-genetica di Vincenzo Consolo. "Il sorriso dell'ignoto marinaio», Madrid, Universidad Complutense de Madrid, «E-Prints Complutense», <http://eprints.ucm.es/8090/>

Nisticò V., 2001, Accadeva in Sicilia. Gli anni ruggenti dell'«Ora» di Palermo, Palermo, Sellerio.

O’Connell D., 2003, “Trista conca': Dantean Anagnorisis and Echo in "Il sorriso dell'ignoto marinaio"», Echi danteschi/ Dantean Echoes, a cura di Roberto Bertoni, Torino, Trauben, p. 85-105.

O'Connell D., 2004, «Il dovere del racconto: Interview with Vincenzo Consolo», The Italianist, 24 (2), p. 238-253.

O’Connell D., 2005, «Zu Luigi: Pirandello and the Sicilian Literary Tradition», Pirandello Studies, 25, p. 29-48.

O'Connell D., 2008, "Consolo narratore e scrittore 'palincestuoso'», Quaderns d'Italià, 13, p. 161-184.

Onofri M., 1995, Tutti a cena da Don Mariano. Letteratura e mafia nella Sicilia della nuova Italia, Milano, Bompiani.

O'rawe C., 2007, «Mapping Sicilian Literature: Place and Text in Bufalino and Consolo», Italian Studies, 62, p. 79-94.

Ovazza M., 1967, Il caso Battaglia. Pascoli e mafia sui Nebrodi, Palermo, Centro di studi ed iniziative culturali Pio La Torre.

Pirandello L., 1973, I vecchi e i giovani, in Tutti i romanzi, II, a cura di Giovanni Macchia e Mario Costanzo, Milano, Mondadori.

Renda F., 1998, Storia della mafia, Palermo, Sigma Edizioni.

Romano S. F., 1952, Momenti del Risorgimento in Sicilia, Firenze, D’Anna.

Santino U., 2000, Storia del movimento antimafia. Dalla lotta di classe all'impegno civile, Roma, Editori Riuniti. 
Segala A. (a cura di), 1988, Littérature Latino-américaine et des Caraïbes $d u$ $\mathrm{XX}^{e}$ siècle. Théorie et pratique de l'édition critique, Roma, Bulzoni.

Segre C., 1991, Intrecci di voci. La polifonia nella letteratura del Novecento, Torino, Einaudi.

Segre C., 2005, Tempo di bilanci. La fine del Novecento, Torino, Einaudi.

Stajano C., 1975, «Il sorriso dell'ignoto marinaio. Due siciliani pazzi per un libro 'unico'», Il Giorno, 30/11/1975.

Tavani G., 1996, «Filologia e genetica», Cuadernos de Filología Italiana, 3, p. 63-90.

Traina G., 2001, Vincenzo Consolo, Fiesole, Cadmo. 